\title{
On Involutions Arising from Graphs
}

\author{
Jurriaan Hage \\ Department of Information and Computing Sciences \\ Universiteit Utrecht, P.O.Box 80.089 \\ 3508 TB Utrecht, The Netherlands \\ jur@cs.uu.nl \\ and \\ Tero Harju \\ Dept. of Mathematics, University of Turku \\ FIN-20014 Turku, Finland \\ e-mail: harju@utu.fi
}

\begin{abstract}
We investigate various aspects of involutions of groups, i.e, anti-automorphisms of order at most two. The emphasis is on finite abelian groups. We count the number of involutions for the cyclic groups, and consider the problem for direct products of groups. We also give a characterization for the set of skewed squares of finitely generated abelian groups with identity as the involution. The present paper is motivated by our research into switching classes of combinatorial graphs where the edges have skew gains.
\end{abstract}

Keywords: finite abelian groups, involutions, cyclic groups, switching classes, graphs

2000 Mathematics Subject Classification: 20K01, 20K30, 05C22

\section{Introduction}

Involutions occur naturally in biological processes especially in the base base inversions of DNA. The material in this paper is mainly motivated not so much by the biological applications but rather by a quest for techniques which enable analysis of networks of processors which can be modelled by actions of groups on finite combinatorial graphs. Such an action is presented by Seidel switching (see $[10,11]$ or $[3,5,12])$, and in a more general setting by switching in the so called skew gain graphs (see $[2,4,6])$.

In the context of Seidel switching (of ordinary graphs), the confronted involution corresponds to the inversion of the underlying group. In the general setting of skew gain graphs more flexibility is gained by having general involutions in the underlying group that acts on the graphs.

In the following we consider involutions in groups without the graphs which employ them. In Section 3, we concentrate on cyclic groups, and then in Section 4 we consider involutions of direct products of finite groups. In Section 5 we 
consider a problem for infinite abelian groups concerning involutions to which Hage [4] showed that the membership problem of switching classes can be reduced.

\section{Preliminaries}

We use $\mathbb{Z}, \mathbb{R}$ and $\mathbb{R}^{+}$to refer to the sets of integers, real numbers and positive real numbers respectively. The cardinality of a set $X$ is denoted by $|X|$. The identity function on $X$ is denoted by $\iota_{X}$, from which the subscript $X$ is omitted if it is clear from the context.

Let $\Gamma$ be a group. For a function $f$, the set of its fixed points is $\operatorname{Fix}(\alpha)=\{a \in$ $\Gamma \mid \alpha(a)=a\}$, and the set of its inverted points is $\operatorname{Inx}(\alpha)=\{a \in \Gamma \mid \alpha(a)=$ $\left.a^{-1}\right\}$.

A bijection $\alpha: \Gamma \rightarrow \Gamma$ is an anti-automorphism, if $\alpha(a b)=\alpha(b) \alpha(a)$ for all $a, b \in \Gamma$. For abelian groups an anti-automorphism is always an automorphism. An anti-automorphism $\alpha$ of the group $\Gamma$ is an involution, if $\alpha^{2}=\iota$, i.e., if $\alpha$ has order at most two. ${ }^{1}$ Let $\operatorname{Inv}(\Gamma)$ denote the set of all involutions of $\Gamma$. We use $\# a$ to denote the order of a group element $a \in \Gamma$. The kernel of a group homomorphism $\alpha: \Gamma \rightarrow \Gamma^{\prime}$ is the set $\operatorname{ker}(\alpha)=\left\{a \mid \alpha(a)=1_{\Gamma^{\prime}}\right\}$. The image of $\alpha$ is $\operatorname{Im}(\alpha)=\left\{b \in \Gamma^{\prime} \mid b=\alpha(a), a \in \Gamma\right\}$. For other standard group notation we refer to Rotman [8].

Example 1. Let $a \in \Gamma$, and let $\delta \in \operatorname{Inv}(\Gamma)$ be an involution. Then $\delta\left(1_{\Gamma}\right)=1_{\Gamma}$, $\delta\left(a^{n}\right)=\delta(a)^{n}$ for integers $n \in \mathbb{Z}$, and $\# \delta(a)=\# a$. The inversion $a \mapsto a^{-1}$ is an involution of each group $\Gamma$.

Clearly, $\delta: \Gamma \rightarrow \Gamma$ is an involution if and only if the mapping $a \mapsto \delta(a)^{-1}$ is an automorphism of order at most two. Therefore, for instance, if $\Gamma$ contains an element $g$ with $\# g=2$, then the mapping $a \mapsto\left(a^{-1}\right)^{g}\left(=g a^{-1} g^{-1}\right)$ is an involution. This is the case among the finite groups $\gamma$ of even order.

The following two results are proven, at least, in [6].

Lemma 1. Let $\delta$ be an involution of a finite group $\Gamma$.

1. Either $\operatorname{Fix}(\delta) \neq\left\{1_{\Gamma}\right\}$, or $\delta$ is the inversion of $\Gamma$ and $\Gamma$ is of odd order.

2. Either $\operatorname{Inx}(\delta) \neq\left\{1_{\Gamma}\right\}$, or $\delta$ is the identity function and $\Gamma$ is an abelian group of odd order.

Recall that the centre of a group $\Gamma$ is the normal subgroup $\mathrm{Z}(\Gamma)=\{x \in \Gamma \mid$ $x y=y x$ for all $y \in \Gamma\}$.

Theorem 1. The centre $Z(\Gamma)$ of $\Gamma$ is closed under every involution of $\Gamma$. In particular, if $\Gamma$ has a nontrivial centre, then for all involutions $\delta$ either $\delta(z)=z$ for all $z \in Z(\Gamma)$ or there exists an element $x \in Z(\Gamma)$ such that $\delta(x)=x^{-1}$ with $x \neq 1_{\Gamma}$.

\footnotetext{
${ }^{1}$ In the literature an element of a group of order two is also called an involution.
} 


\section{Involutions of cyclic groups}

Each finite cyclic group is isomorphic to $\mathbb{Z}_{n}$ for some $n$, and by the fundamental theorem of abelian groups, it can be written as a direct sum

$$
\mathbb{Z}_{n} \cong \mathbb{Z}_{p_{1}^{m_{1}}} \oplus \mathbb{Z}_{p_{2}^{m_{2}}} \oplus \cdots \oplus \mathbb{Z}_{p_{r}^{m_{r}}}
$$

of cyclic groups $\mathbb{Z}_{p_{i}^{m_{i}}}$, where $p_{i} \geq 2$ are distinct prime numbers such that $n=$ $p_{1}^{m_{1}} p_{2}^{m_{2}} \ldots p_{r}^{m_{r}}$.

Let $\delta \in \operatorname{Inv}\left(\mathbb{Z}_{n}\right)$, and suppose $\delta(1)=k$. Now, $\delta(i) \equiv i k(\bmod n)$ and so $1=\delta(k) \equiv k^{2}(\bmod n)$, i.e.,

$$
k^{2} \equiv 1 \quad(\bmod n)
$$

Example 2. Let then $n=16$. If (1) holds for $k$ then it also holds for $n-k$, so $k=15$ is also possible. It is easy to see that if $k>1$ then $k \geq \sqrt{n+1}$ and hence $k$ cannot be equal to 2,3 or 4 . From Example 1 we know that $\# 1=\# k$ and hence $k$ generates $\mathbb{Z}_{n}$ as well, implying $(n, k)=1$. This means that other possible values for $k$ that have to be examined are 5 and 7 . Of these only 7 works (and thus also 9).

Let $\xi: \mathbb{N} \rightarrow\{-1,0,1\}$ be defined by

$$
\xi(n)= \begin{cases}1 & \text { if } 8 \mid n \\ -1 & \text { if } 2 \mid n \text { and } 4 \not x \\ 0 & \text { otherwise }\end{cases}
$$

Lemma 2. If $n=p^{m}$ for a prime $p$ and $m \in \mathbb{N}$ then (1) has exactly $2^{1+\xi(n)}$ solutions $k$ with $1 \leq k \leq n-1$.

Proof. The two solutions $k=1$ and $k=n-1$ work for any cyclic group. In the case $p=2$ and $m=1$ these give the same unique solution.

Assume that (1) holds for $k \notin\{1, n-1\}$. Now, $p^{m} \mid(k-1)(k+1)$, and thus $p=2$; otherwise $p^{m}=k+1$ (because $k<n$ ) and so $k=n-1$. Suppose $2^{i} \mid k-1$ and $2^{j} \mid k+1$ with $i+j=m$. We have $0 \leq i<m$.

If $i=0$ and thus $j=m$ then $n=k+1$; a contradiction. Suppose then that $i>0$ in which case also $j>0$. Let $r=\min (i, j)$. Then $2^{r} \mid(k+1)-(k-1)=2$, i.e., $r=1$. If $r=i$, then $j=m-1$, and so $2^{m-1} \mid k+1$. If $r=j$, then $i=m-1$, and so $2^{m-1} \mid k-1$. Hence, in both cases,

$$
k \equiv \pm 1 \quad \bmod 2^{m-1} .
$$

For $m=1$ and $m=2$, we obtain the same solutions as in the above, and for $m \geq 3$, we have two solutions.

Summarizing, we have one solution if $p=2$ and $m=1$, four solutions if $p=2$ and $m \geq 3$ and two if $p \neq 2$ or $p=2$ and $m=2$.

In the above we have only proven half of what we need to prove, namely indicating possible solutions. The fact that these solutions do indeed always exist can be verified easily from (1).

Hence, for every $n$ the number of solutions equals $2^{1+\xi(n)}$. 
Theorem 2. Let $n=p_{1}^{m_{1}} p_{2}^{m_{2}} \ldots p_{r}^{m_{r}}$ be a prime decomposition of $n$ with $p_{i}<$ $p_{i+1}$, and $m_{i}>0$ for $1 \leq i \leq r-1$. Then $\left|\operatorname{Inv}\left(\mathbb{Z}_{n}\right)\right|=2^{r+\xi(n)}$.

Proof. Lemma 2 can be applied to each $p_{j}^{m_{j}}$, with $1 \leq j \leq r$. We can then use Theorem 122 in the book of Hardy and Wright [7] to conclude that the total number of solutions equals the product of the numbers of solutions to the separate equations for $p_{j}^{m_{j}}$ for $1 \leq j \leq r$.

If $p_{1} \neq 2$ then every prime number gives two solutions yielding a total of $2^{r}$ solutions, and indeed in this case $\xi(n)=0$. If $p_{1}=2$ then we have three possibilities: $m_{1}=1$ and hence $p_{1}$ yields only one solution and hence we have a total of $2^{r-1}$ solutions. The other two cases, $m_{1}=2$ and $m_{1}>2$, follow similarly, because now $\xi(n)=\xi\left(p_{1}^{m_{1}}\right)$.

Note that the involutions can be found by solving two sets of equations using the Chinese Remainder Theorem, see [7].

\section{Involutions of group products}

In the previous section it turned out that finding involutions of cyclic groups is rather easy. In this section it is shown that computing the involutions of a direct product of two groups involves taking the cartesian product of the sets of involutions of both groups.

We remind, see, e.g., Rotman [8], that the inner and outer direct products of groups coincide up to isomorphism, i.e., for a group $\Gamma$, if $\Gamma_{1}$ and $\Gamma_{2}$ are two of its normal subgroups such that $\Gamma=\Gamma_{1} \Gamma_{2}$ and $\Gamma_{1} \cap \Gamma_{2}=\left\{1_{\Gamma}\right\}$, then $\Gamma \cong \Gamma_{1} \times \Gamma_{2}$. If $\Gamma=\Gamma_{1} \Gamma_{2}$ is a direct product, then $a_{1} a_{2}=a_{2} a_{1}$ for all $a_{1} \in \Gamma_{1}$ and $a_{2} \in \Gamma_{2}$, and, moreover, each element $a \in \Gamma$ has a unique representation as a product $a=a_{1} a_{2}$, where $a_{i} \in \Gamma_{i}$.

Let $\Gamma=\Gamma_{1} \Gamma_{2}$ be a direct product, and let $\alpha: \Gamma \rightarrow \Gamma$ be any function. We define the projections $\alpha^{(i)}: \Gamma \rightarrow \Gamma_{i}$ for $i=1,2$ by: for each $a \in \Gamma$ let $\alpha(a)=$ $\alpha^{(1)}(a) \alpha^{(2)}(a)$, where $\alpha^{(1)}(a) \in \Gamma_{1}$ and $\alpha^{(2)}(a) \in \Gamma_{2}$. By the uniqueness property of direct products, these functions are well defined. We also write

$$
\delta^{[i]}: \Gamma_{i} \rightarrow \Gamma_{i}
$$

for the restriction of $\delta^{(i)}$ onto the subgroup $\Gamma_{i}$ for $i=1,2$.

The following example shows that an involution of a direct product cannot necessarily be obtained by projections of its components.

Example 3. Let $\Gamma=\Gamma_{1} \times \Gamma_{1}$ for a group $\Gamma_{1}$, and let $\delta$ be the reversed inversion on $\Gamma$, that is,

$$
\delta\left(a_{1}, a_{2}\right)=\left(a_{2}^{-1}, a_{1}^{-1}\right)
$$

for all $a_{1}, a_{2} \in \Gamma_{1}$. Then $\delta$ is an involution of $\Gamma$. Indeed, it is clear that $\delta^{2}=\iota$, and, moreover, for all $a_{i}, b_{i} \in \Gamma_{1}$,

$$
\begin{aligned}
\delta\left(a_{1}, a_{2}\right) \cdot \delta\left(b_{1}, b_{2}\right) & =\left(a_{2}^{-1}, a_{1}^{-1}\right)\left(b_{2}^{-1}, b_{1}^{-1}\right)=\left(a_{2}^{-1} b_{2}^{-1}, a_{1}^{-1} b_{1}^{-1}\right) \\
& =\left(\left(b_{2} a_{2}\right)^{-1},\left(b_{1} a_{1}\right)^{-1}\right)=\delta\left(b_{1} a_{1}, b_{2} a_{2}\right) \\
& =\delta\left(\left(b_{1}, b_{2}\right) \cdot\left(a_{1}, a_{2}\right)\right) .
\end{aligned}
$$


However, $\delta$ is not of the form $\delta=\left(\delta_{1}, \delta_{2}\right)$ for any functions (let alone involutions) $\delta_{1}$ and $\delta_{2}$ of $\Gamma_{1}$, if $\Gamma_{1}$ is nontrivial.

However, we do have

Theorem 3. Let $\Gamma=\Gamma_{1} \Gamma_{2}$ be a direct product.

1. If $\delta_{i} \in \operatorname{Inv}\left(\Gamma_{i}\right)$ for $i=1,2$, then the function $\delta: \Gamma \rightarrow \Gamma$ defined by

$$
\delta(a)=\delta_{1}\left(a_{1}\right) \delta_{2}\left(a_{2}\right) \quad \text { for } a=a_{1} a_{2} \text { with } a_{i} \in \Gamma_{i}
$$

is an involution of $\Gamma$.

2. If $\delta \in \operatorname{Inv}(\Gamma)$, then there are normal subgroups $\Delta_{1}$ and $\Delta_{2}$ of $\Gamma$ such that $\Gamma=\Delta_{1} \Delta_{2}$ is a direct product with $\left|\Delta_{1}\right|=\left|\Gamma_{1}\right|,\left|\Delta_{2}\right|=\left|\Gamma_{2}\right|$ for which $\delta^{[i]}: \Delta_{i} \rightarrow \Delta_{i}$ is an involution of $\Delta_{i}$ for $i=1,2$.

Proof. In order to prove (i), let $\delta_{i}$ be involutions as stated. Let $a=a_{1} a_{2}$ and $b=b_{1} b_{2}$ for $a_{1}, b_{1} \in \Gamma_{1}$ and $a_{2}, b_{2} \in \Gamma_{2}$. Now, for the function $\delta$ as defined in the claim,

$$
\begin{aligned}
\delta(a b) & \left.=\delta\left(a_{1} a_{2} b_{1} b_{2}\right)\right)=\delta\left(a_{1} b_{1} a_{2} b_{2}\right)=\delta_{1}\left(a_{1} b_{1}\right) \delta_{2}\left(a_{2} b_{2}\right) \\
& =\delta_{1}\left(b_{1}\right) \delta_{1}\left(a_{1}\right) \delta_{2}\left(b_{2}\right) \delta_{2}\left(a_{2}\right)=\delta_{1}\left(b_{1}\right) \delta_{2}\left(b_{2}\right) \delta_{1}\left(a_{1}\right) \delta_{2}\left(a_{2}\right) \\
& =\delta\left(b_{1} b_{2}\right) \delta\left(a_{1} a_{2}\right)=\delta(b) \delta(a)
\end{aligned}
$$

and thus $\delta$ is an anti-automorphism of $\Gamma$. Further, the condition $\delta^{2}(a)=a$ is easily checked.

For (ii) suppose first that $\delta \in \operatorname{Inv}(\Gamma)$, and define

$$
\Delta_{1}=\left\{\delta(a) \mid a \in \Gamma_{1}\right\} \text { and } \Delta_{2}=\left\{\delta(b) \mid b \in \Gamma_{2}\right\} .
$$

Clearly, $a \in \Delta_{1}$ (resp. in $\Delta_{2}$ ) if and only if $\delta(a) \in \Gamma_{1}\left(\operatorname{resp} . \delta(a) \in \Gamma_{2}\right)$. Since an involution is a bijection, we have immediately that $\left|\Delta_{i}\right|=\left|\Gamma_{i}\right|$ for $i=1,2$.

We show then that $\Delta_{1}$ and $\Delta_{2}$ are normal subgroups of $\Gamma$. Indeed, let $y=$ $a u a^{-1}$ for some $a \in \Gamma$ and $u \in \Delta_{1}$. Now, $\delta(y)=\delta(a)^{-1} \delta(u) \delta(a) \in \Gamma_{1}$, since $\delta(u) \in \Gamma_{1}$ and $\Gamma_{1}$ is a normal subgroup of $\Gamma$. This shows that $\Delta_{1}$ is normal in $\Gamma$. The case for $\Delta_{2}$ is symmetric.

Next we observe that $\Delta_{1} \cap \Delta_{2}=\left\{1_{\Gamma}\right\}$ is the trivial subgroup of $\Gamma$. Furthermore, if $a \in \Gamma$, then $a=a_{2} a_{1}$ for some $a_{i} \in \Gamma_{i}$, because $\Gamma=\Gamma_{2} \Gamma_{1}$. Therefore, $\delta(a)=\delta\left(a_{1}\right) \delta\left(a_{2}\right)$, where $\delta\left(a_{1}\right) \in \Delta_{1}$ and $\delta\left(a_{2}\right) \in \Delta_{2}$. Since each element $b \in \Gamma$ is an image $b=\delta(a)$, we have shown that $\Gamma=\Delta_{1} \Delta_{2}$ is a direct product of $\Gamma$.

It is clear that $\delta^{[i]}$ is an involution of $\Delta_{i}$ for both $i=1$ and $i=2$.

In particular, if $\Gamma$ is an abelian group, then it is a direct product (sum) of cyclic groups, and thus Theorem 4 states that the involutions of an abelian group can be obtained from the cyclic groups $\mathbb{Z}_{p^{k}}$ that are its direct components. However, counting the number of involutions of $\Gamma$ is not reduced in this way to the number of involutions of its direct components, because part (ii) of Theorem 4 uses 'swappings' of subgroups. 
Example 4. Let $\Gamma=\mathbb{Z}_{2} \oplus \mathbb{Z}_{2}$. The groups $\Gamma$ and $\mathbb{Z}_{2}$ have only the identity function $\iota$ as their involution (equal to the group inversion), but in the case of the former, it is not the only one. Indeed, the following swapping function $\delta$ is an involution of $\Gamma$ :

$$
\delta((a, b))=(b, a) \text { for } a, b \in\{0,1\} .
$$

\section{The set $\Delta^{2}(\Gamma, \delta)$}

In [4] the following set of decomposable values arose (called the skewed squares in [5]): for a group $\Gamma$ and its inversion $\delta$, let

$$
\Delta^{2}(\Gamma, \delta)=\{a \in \Gamma \mid a=b \delta(b) \text { for some } b \in \Gamma\} .
$$

In this section we investigate this set in more detail.

First, in the following proof, we have a connection of the above set with decompositions of abelian $p$-groups.

Theorem 4. Let $\Gamma$ be a finite abelian group of odd order, and let $\delta \in \operatorname{Inv}(\Gamma)$. Then $\Gamma$ is isomorphic to the direct sum $\operatorname{Fix}(\delta) \times \operatorname{Inx}(\delta)$.

Proof. It is well known that in each abelian group of odd order every element $a \in \Gamma$ has a unique "square root" $x$ in $\Gamma$, i.e., $x^{2}=a$, when adopting the multiplicative notation; see Rotman [9, Page 81]. Now, for each $a \in \Gamma, a=x y$ holds for some $x \in \operatorname{Fix}(\delta)$ and $y \in \operatorname{Inx}(\delta)$ if and only if $a^{-1} x=y^{-1}=\delta(y)=$ $\delta(a) \delta\left(x^{-1}\right)=\delta(a) x^{-1}$ if and only if $x^{2}=a \delta(a)$ and $y^{2}=(a \delta(a))^{-1}$. This proves the claim since $\operatorname{Fix}(\delta) \cap \operatorname{Inx}(\delta)=\left\{1_{\Gamma}\right\}$.

Example 5. If $\delta$ is the group inversion, then clearly $\Delta^{2}(\Gamma, \delta)=\left\{1_{\Gamma}\right\}$. Also, it is easy to determine that $\Delta^{2}(\mathbb{Z}, \iota)$ is the set of even numbers, $\Delta^{2}(\mathbb{R}, \iota)=\mathbb{R}$ for the additive group of reals, and $\Delta^{2}\left(\mathbb{R}^{+}, \iota\right)=\mathbb{R}^{+}$for the multiplicative group of positive real numbers.

Given a fixed group $\Gamma$ with an involution $\delta$, we define the function $s_{\Gamma, \delta}$ by

$$
s_{\Gamma, \delta}(a)=a \delta(a) \text { for } a \in \Gamma
$$

so that we have $\operatorname{Im}\left(s_{\Gamma, \delta}\right)=\Delta^{2}(\Gamma, \delta)$. When $\Gamma$ and $\delta$ are obvious from the context we write $s$ instead of $s_{\Gamma, \delta}$.

Lemma 3. For any group $\Gamma, \Delta^{2}(\Gamma, \delta)$ is closed under the group inversion, and $\Delta^{2}(\Gamma, \delta) \subseteq \operatorname{Fix}(\delta)$.

Proof. Let $s(a) \in \Delta^{2}(\Gamma, \delta)$. Then $s(a)^{-1}=\delta(a)^{-1} a^{-1}=s\left(\delta(a)^{-1}\right) \in \Delta^{2}(\Gamma, \delta)$. For the second part, $\delta(s(a))=\delta(a \delta(a))=a \delta(a)=s(a)$. 
Because involutions of a direct product do not always project onto the factors it is unlikely that we can determine the skewed squares of a group $\Gamma$ with involution $\delta$ from the skewed squares of the groups in a decomposition of $\Gamma$, see Example 3. However, we do have that involutions $\delta_{1}$ and $\delta_{2}$ of groups $\Gamma_{1}$ and $\Gamma_{2}$, respectively, can be used to construct an involution $\left(\delta_{1}, \delta_{2}\right)$ for $\Gamma_{1} \times \Gamma_{2}$ by applying them componentwise. For involutions thus constructed the set of skewed squares can be constructed from the sets of skewed squares of the factors as proved by the following result.

Theorem 5. Let $\Gamma=\Gamma_{1} \times \Gamma_{2}$ be a direct product of groups and let $\delta_{i} \in \operatorname{Inv}\left(\Gamma_{i}\right)$ for $i=1,2$. Then $\Delta^{2}\left(\Gamma,\left(\delta_{1}, \delta_{2}\right)\right)=\Delta^{2}\left(\Gamma_{1}, \delta_{1}\right) \times \Delta^{2}\left(\Gamma_{2}, \delta_{2}\right)$.

Proof. It holds that

$$
(a, b)\left(\delta_{1}, \delta_{2}\right)(a, b)=(a, b)\left(\delta_{1}(a), \delta_{2}(b)\right)=\left(a \delta_{1}(a), b \delta_{2}(b)\right),
$$

where $a \delta_{1}(a) \in \Delta^{2}\left(\Gamma_{1}, \delta_{1}\right)$ and $b \delta_{2}(b) \in \Delta^{2}\left(\Gamma_{2}, \delta_{2}\right)$.

\section{Example: The case of the identity involution}

In the rest of the article we assume that $\delta$ is the identity function, $\iota$. Note that $\iota$ is an involution of every abelian group and no other, so $\Gamma$ must be abelian. Written additively, the definition of $\Delta^{2}$ reduces to

$$
\{a \in \Gamma \mid a=2 b \text { for some } b \in \Gamma\} .
$$

So in a sense $\Delta^{2}$ contains the "even elements" of the group $\Gamma$.

Example 6. We can easily verify that $\Delta^{2}\left(\mathbb{Z}_{2}, \iota\right)=\{0\}, \Delta^{2}\left(\mathbb{Z}_{3}, \iota\right)=\{0,1,2\}$, $\Delta^{2}\left(\mathbb{Z}_{4}, \iota\right)=\{0,2\}$, and $\Delta^{2}\left(\mathbb{Z}_{6}, \iota\right)=\{0,2,4\}$.

From this example it emerges that for even $n, \Delta^{2}\left(\mathbb{Z}_{n}, \iota\right)$ contains exactly the even numbers and for odd $n$, it equals the entire $\Gamma$. The latter is not surprising since if $x \in \Gamma$ has order $n$ and $(m, n)=1$ then $x$ is divisible by $m$.

It is plain that $\Delta^{2}\left(\mathbb{Z}_{2^{k}}, \iota\right)=\left\{0,2,4,6, \ldots, 2^{k-1}\right\}$, for $k \geq 1$, and $\Delta^{2}\left(\mathbb{Z}_{p^{k}}, \iota\right)=$ $\mathbb{Z}_{p^{k}}$ where $p>2$ is prime and $k \geq 1$, and $\Delta^{2}(\mathbb{Z}, \iota)$ contains the even numbers and so by Theorem 5 and the fundamental theorem of finitely generated abelian groups we get the following result.

Theorem 6. Let $\Gamma$ be a finitely generated abelian group with a decomposition $\Gamma_{1} \oplus \ldots \oplus \Gamma_{n}$ (unique up to the order of the summands) into infinite cyclic groups and cyclic p-groups. Then $\Delta^{2}(\Gamma, \iota)=\Delta^{2}\left(\Gamma_{1}, \iota\right) \oplus \ldots \oplus \Delta^{2}\left(\Gamma_{\ell}, \iota\right)$.

The previous theorem says nothing about abelian groups that are not finitely generated such as $\mathbb{R}^{+}$under multiplication. To deal with $\mathbb{R}^{+}$, we recall the notion of divisibility: a group is divisible if for every element $a$ and $n>0$, we have $a=b^{n}$ for some $b$. Note that the set $\Delta^{2}$ is only concerned with divisibility by two. Therefore, the following result is easy. 
Theorem 7. If $\Gamma$ is divisible then $\Delta^{2}(\Gamma, \iota)=\Gamma$.

A result such as this suggests that it may be worthwhile to investigate other decompositions of groups, into divisible and reduced components, or into torsion and torsion free components.

Acknowledgement. We thank professor R. Tijdeman and W. Kosters for discussions on the involutions of cyclic groups.

\section{References}

1. D. G. Corneil and R. A. Mathon, editors. Geometry and Combinatorics: Selected Works of J. J. Seidel. Academic Press, Boston, 1991.

2. A. Ehrenfeucht, J. Hage, T. Harju, and G. Rozenberg. Embedding in switching classes with skew gains. In H. Ehrig, G. Engels, F. Parisi-Presicce, and G. Rozenberg, editors, Graph Transformations, Second International Conference, ICGT 2004, volume LNCS 3256, pages 257 - 270. Springer Verlag, September/October 2004.

3. A. Ehrenfeucht, T. Harju, and G. Rozenberg. The Theory of 2-Structures. World Scientific, Singapore, 1999.

4. J. Hage. The membership problem for switching classes with skew gains. Fundamenta Informaticae, 39(4):375-387, 1999.

5. J. Hage. Structural Aspects Of Switching Classes. PhD thesis, Leiden Institute of Advanced Computer Science, 2001. http://www.cs.uu.nl/people/jur/2s.html.

6. J. Hage and T. Harju. The size of switching classes with skew gains. Discrete Math., 215:81 - 92, 2000.

7. G. H. Hardy and E. M. Wright. An Introduction to the Theory of Numbers. Oxford Science Publications, Hong Kong, 5th edition, 1983.

8. J. J. Rotman. The Theory of Groups. Allyn and Bacon, Boston, 2nd edition, 1973.

9. J. J. Rotman. Advanced Modern Algebra. Pearson Education, Inc., Upper Saddle River, 2002.

10. J. J. Seidel. A survey of two-graphs. In Intern. Coll. Teorie Combinatorie (Roma,1973), volume I, pages 481-511, Rome, 1976. Accad. Naz. Lincei. Reprinted in [1].

11. J. J. Seidel and D. E. Taylor. Two-graphs, a second survey. In L. Lovasz and V.T. Sós, editors, Algebraic Methods in Graph Theory (Proc. Internat. Colloq., Szeged, 1978), volume II, pages 689-711, Amsterdam, 1981. North-Holland. Reprinted in [1].

12. T. Zaslavsky. Biased graphs. I. Bias, balance, and gains. J. Combin. Theory, Ser. $B, 47: 32-52,1989$. 